\title{
Una asignatura universitaria para el desarrollo de las competencias personales transversales
}

\author{
Paula Crespí Rupérez a , José Manuel García Ramos ${ }^{\text {b }}$ \\ ap.crespi.prof@ufv.es, Facultad de Educación. Universidad Francisco de Vitoria. Madrid (España) ${ }^{\mathrm{b}}$ \\ jmgramos@ccedu.ucm.es, Facultad de Educación. Universidad Complutense de Madrid (España)
}

\begin{abstract}
Resumen
Hoy en día es una realidad el hecho de que nuestros alumnos necesitan desarrollar competencias transversales de cara a poder cumplir con su vocación y misión en su vida, ya sea en el ámbito personal, social, académico o empresarial. En este sentido, el Espacio Europeo de Educación Superior (EEES) recoge la necesidad de que la educación superior forme en competencias genéricas (no sólo específicas). También la empresa demanda cada vez más que los empleados tengan desarrolladas ciertas habilidades sociales o blandas (soft skills) antes de contratarles o promocionarles. Sin embargo, no parece que el sistema educativo, en concreto, el referente a la educación superior, ofrezca a sus alumnos programas o asignaturas diseñadas ad hoc para el desarrollo de estas competencias transversales tan críticas para el EEES y tan demandas en el ámbito empresarial; y en la vida en general. Es por esto que, nuestro estudio ofrece una propuesta innovadora de asignatura, impartida con una metodología experiencial por docentes expertos en esta materia, cuya finalidad es el desarrollo de dichas competencias transversales y su transferencia a la realidad: personal, social, académica y profesional.
\end{abstract}

Palabras clave: Competencias genéricas, educación superior, habilidades blandas, programa de desarrollo de competencias transversales

\section{Introducción}

La universidad Francisco de Vitoria, conectando con el origen y misión de toda universidad, pretende formar una auténtica comunidad universitaria que, por su formación y liderazgo, promuevan la generación de una sociedad sustentada por la búsqueda continua de la verdad y del bien (Nuestra misión hoy, 2016). "Una comunidad de buscadores de la 
Habilidades y Competencias de la Persona. Una asignatura para el desarrollo de las competencias personales

verdad, de la verdad sobre el hombre, sobre la realidad y sobre el sentido último de la vida" (Crespí, 2018 p.31). En definitiva, un comunidad que, siguiendo a autores como García Ramos (1991,2017), Jaspers (2013), Moncada (2008), Ortega y Gasset (2004) y Pérez-Díaz (2010):

- $\quad$ Favorezca el desarrollo de la dignidad del hombre.

- $\quad$ Ponga al alumno en búsqueda de su propia vocación y proyecto vital.

- $\quad$ Facilite la formación integral del alumno, dirigida a su pleno desarrollo.

Una universidad que anhela hacer crecer a toda la persona, que es el alumno, y que quiere prepararle lo mejor posible para su vida (personal, académica y profesionalmente). En este sentido, pretendemos ofrecer una formación que atienda individualmente a cada alumno, de modo que este pueda dar respuesta a su propia vida; convirtiéndose a su vez en una persona excelente (en cualquier ámbito: personal, social, académico, profesional).

Por tanto, queremos ofrecer a nuestros alumnos una formación integral, dirigida al pleno desarrollo de todo su ser. En este sentido, nos unimos a la reflexión del EEES y del ámbito empresarial y entendemos que los planes de estudio han de ofrecer una formación específica en competencias transversales (competencias genéricas para el EEES y soft skills, para la empresa) además de en competencias técnicas (Alles, 2008; Benito y Cruz, 2006; González Ariza, 2017; Gutiérrez Tobar, 2010; Villardón-Gallego, 2015).

En este sentido, la UFV plantea la materia de Habilidades y Competencias de la Persona. Esta asignatura, a través del desarrollo de las competencias personales, especialmente las transversales, pretende acompañar a sus alumnos en la búsqueda de su vocación y misión, de modo que cada uno de ellos pueda llegar a ser quién está llamado a ser.

\section{Metodología}

\subsection{La asignatura de HCP (Habilidades y Competencias de la Persona)}

Dentro del plan de formación integral, la UFV, ofrece la asignatura de HCP. Esa asignatura es común para todos los alumnos de primero, independientemente de su titulación. HCP, pretende, dentro del abanico de formación integral, acompañar al alumno en el desarrollo de sus competencias personales, fundamentalmente las transversales, para ponerle en su propio camino de madurez, excelencia y plenitud (Crespí, 2018).

Hablamos de competencia personal como "el conjunto dinámico de conocimientos (saber), habilidades o destrezas (saber hacer), actitudes y valores (saber ser) que, interiorizados y encarnados en nuestros actos, comportamientos o maneras de hacer, nos 
ponen en el camino de nuestra propia madurez, excelencia, plenitud y felicidad" (Crespí, 2019. p. 98).

HCP pretende desarrollar especialmente las competencias transversales, aquellas que están relacionadas con un desempeño excelente en algún área de nuestra vida (personal o social; académico o profesional). En la educación superior reciben el nombre de genéricas y en el ámbito empresarial, el de soft skills. Por tanto, aunque también alude, de manera indirecta a las competencias técnicas, aquellas que están relacionadas con desempeño excelente ante una determinada actividad relativa a un área o tema específico, no son estas su principal objeto de desarrollo, sino que lo son del resto de asignaturas técnicas y específicas del grado. En la educación superior reciben el nombre de específicas y en el ámbito empresarial, el de hard skills (Crespí y García Ramos, 2019).

HCP es una asignatura que está claramente centrada en el alumno (no en los contenidos), y más concretamente en su desarrollo personal, ya que pretende que los alumnos aumenten su nivel competencial en relación a las competencias transversales críticas que le ofrece esta materia. Emplea una metodología dialógica, para que el alumno se ponga en verdadera búsqueda, y experiencial, para que pueda ser transferible a su realidad. Por último, esta asignatura requiere de un formador experto en el desarrollo de las competencias personales transversales y que se mantenga en la incesante búsqueda de la excelencia y plenitud personal.

Esta asignatura pretende desarrollar específicamente las competencias:

- Intrapersonales, aquellas principalmente de "relación con uno mismo", como "la proactividad, la mirada profunda, el conocimiento y superación personal y la búsqueda de sentido" (Crespí, 2018, p. 202).

- Interpersonales, aquellas principalmente de relación con el otro, como "el trabajo en equipo, la comunicación, la resolución de conflictos-negociación y el liderazgo" (Crespí, 2018, p. 202).

- Cognitivas, aquellas principalmente relacionadas con nuestra capacidad de pensar, como la gestión del tiempo y proyectos y la estrategia. Estas competencias están al servicio de las otras dos; es decir HCP pretende que el alumno las desarrolle en tanto que son necesarias para el desarrollo de las intra e interpersonales (Crespí, 2019).

\section{2. Ámbitos de desarrollo de HCP}

Para provocar el desarrollo de estas competencias, HCP, se configura en dos ámbitos complementarios (Crespí, 2018): 

personales

La mentoría: ámbito de encuentro, en el que el alumno, acompañado individualmente por su mentor, desarrolla especialmente las competencias personales intrapersonales, y subsidiariamente las cognitivas. El programa de mentoría cuenta con 6 encuentros individuales y presenciales de una hora de duración cada uno. Estos están distribuidos a lo largo del curso (tres en el primer cuatrimestre y otros tres en el segundo). Existe un programa concreto para cada mentoría, que pasamos a detallar:

En la primera mentoría las competencias principales objeto de desarrollo son: mirada profunda y proactividad. Se pretende que el alumno empiece a verse como el protagonista de su propia vida y descubra el valor transformador de la mirada profunda respecto de sí mismo, de los demás y de la realidad.

En la segunda mentoría la competencia principal objeto de desarrollo es el conocimiento personal. Se pretende que el alumno empiece a conocerse mejor. Para ello, se aplican diversas técnicas que le ayudan en esta tarea como el análisis DAFO y el test de caracterología; todo ello con la finalidad de que empiece a descubrir su propia personalidad, forjada a su vez de temperamento y carácter.

En la tercera mentoría las competencias principales objeto de desarrollo son: aceptación y superación personal. Se pretende que el alumno entienda que no todos los caminos conducen a la excelencia y plenitud personal; y en este, la importancia de los hábitos creativos.

En la cuarta mentoría la competencia principal objeto de desarrollo es nuevamente el conocimiento personal; pero desde una perspectiva más profunda e interpelante que sitúa al alumno como alguien único e irrepetible para los demás: familia, universidad, amigos, futuro trabajo, etc. (don para el otro).

En la quinta mentoría la competencia principal objeto de desarrollo es la búsqueda de sentido. Se pretende que el alumno empiece a dar respuesta a su vocación; y en tanto que esta, a su visión y misión.

En la sexta mentoría se recogen todas las anteriores a través de la técnica del portafolio, donde el alumno ha de presentar con sentido global una recopilación de todas las mentorías y competencias trabajadas, presentando todos sus trabajos autónomos.

La nota de la mentoría supone el $25 \%$ de la nota final de HCP.

El aula: ámbito de encuentro en el que el estudiante, acompañado por su formador y sus compañeros, desarrolla especialmente las competencias de carácter interpersonal, y subsidiariamente las cognitivas. Se pretende que el aula (profesor y alumnos), partiendo del trabajo en equipo y con una adecuada comunicación, pueda llegar a convertirse en una verdadera comunidad de aprendizaje. 
Para ello, los alumnos trabajan en equipo desde el primer día de clase, con el objetivo de poner en marcha un proyecto transversal que, tratando un tema técnico (propio de su titulación), implique una mejora transformacional de la sociedad en la que viven.

Este proyecto recibe el nombre de transversal porque:

1. Un primer objetivo es lograr que sea un espacio real de aprendizaje de las competencias trasversales objeto de la asignatura: interpersonales (trabajadas más en el aula), intrapersonales (trabajadas más en la mentoría) y cognitivas (aula y mentoría).

2. Un segundo objetivo es generar una mejora transformacional que les permita integrar, por un lado, las competencias técnicas que les ofrecen las otras asignaturas de su grado, y por otro, las competencias transversales objeto de HCP.

Este proyecto implica las siguientes fases:

1.- Ponerse de acuerdo por consenso sobre qué idea y tema quieren trabajar, así como qué quieren mejorar en la sociedad. Esta fase pone en juego principalmente la competencia de toma de decisión, escucha activa y mirada profunda.

2.- Llevar a cabo la decisión tomada, de modo que se produzca una verdadera mejora en la realidad. Han de medir también el impacto real (cuantitativa y cualitativamente). Esta fase pone en juego principalmente las competencias de proactividad, búsqueda de sentido, gestión del tiempo y proyecto, comunicación, resolución de conflictos y trabajo en equipo.

3.- Presentar una memoria escrita de su trabajo y la defensa oral del mismo ante un tribunal experto tanto en competencias técnicas (profesores y miembros de la dirección de su grado) como en competencias transversales (profesor de HCP y mentores). Esta fase pone en juego principalmente las competencias de proactividad, gestión del tiempo, comunicación y trabajo en equipo.

El trabajo que realizan en el aula computa un $75 \%$ de la nota final de HCP.

En cualquier caso, en HCP (aula-mentoría) es importante poder generar un encuentro con el alumno para poder acompañarle adecuadamente, como individuo o como miembro de un equipo de trabajo, en su propio desarrollo personal y búsqueda de la excelencia y plenitud a la que están llamados.

\section{Resultados}

La siguiente tabla nos muestra los resultados cuantitativos de la evaluación de HCP, aula y mentoría, en relación a los resultados globales de la UFV, para el año académico 20182019. 
Habilidades y Competencias de la Persona. Una asignatura para el desarrollo de las competencias personales

Tabla 1. Resultados del CEDA: cuestionario sobre la actividad docente

\begin{tabular}{|c|c|c|c|c|c|c|c|c|c|c|c|c|}
\hline \multirow{3}{*}{ Núcleo } & \multirow{3}{*}{\multicolumn{2}{|c|}{ ftem }} & \multirow{2}{*}{\multicolumn{4}{|c|}{$\begin{array}{l}\text { MEDIA AULA HCP } \\
\text { N: } 1.489\end{array}$}} & \multirow{2}{*}{\multicolumn{4}{|c|}{$\begin{array}{l}\text { MEDIA MENTORIA HCP } \\
\text { N: } 1.443\end{array}$}} & \multirow{2}{*}{\multicolumn{2}{|c|}{$\begin{array}{l}\text { MEDIA UFV } \\
\mathrm{N}: 63.377\end{array}$}} \\
\hline & & & & & & & & & & & & \\
\hline & & & \multirow{3}{*}{\begin{tabular}{|c|c|} 
Núcleo & \\
4,53 & \\
\end{tabular}} & Item & D.T. & Moda & Núcleo & Item & D.T. & Moda & Núcleo & Item \\
\hline \multirow{2}{*}{$\begin{array}{c}\text { Programación- } \\
\text { Organización de la } \\
\text { Enseñanza }\end{array}$} & 1 & $\begin{array}{l}\text { "Tengo suficientemente claro la planificación inicial de la asignatura (actividades, } \\
\text { criterios evaluación)". }\end{array}$ & & 4,55 & 1,35 & 5 & \multirow{2}{*}{5,11} & 5,15 & 1,09 & 6 & \multirow{2}{*}{4,67} & 4,73 \\
\hline & 2 & "La metodologia de enseñanza de la asignatura favorece mi aprendizaje". & & 4,5 & 1,41 & 6 & & 5,07 & 1,11 & 6 & & 4,60 \\
\hline \multirow{2}{*}{$\begin{array}{l}\text { Dominio de } \\
\text { Contenido }\end{array}$} & 3 & "Las explicaciones sobre el contenido de la materia me resultan claras". & \multirow{2}{*}{4,79} & 4,68 & 1,32 & 6 & \multirow{2}{*}{5,27} & 5,21 & 1,05 & 6 & \multirow{2}{*}{4,84} & 4,70 \\
\hline & 4 & "Las dudas planteadas me son resueltas". & & 4,91 & 1,25 & 6 & & 5,34 & 0,99 & 6 & & 4,97 \\
\hline \multirow{2}{*}{$\begin{array}{c}\text { Innovación Docente. } \\
\text { Motivación } \\
\end{array}$} & 5 & "Puedo conectar la asignatura con situaciones de la vida real". & \multirow{2}{*}{4,55} & 4,76 & 1,35 & 6 & \multirow{2}{*}{5,00} & 5,19 & 1,18 & 6 & \multirow{2}{*}{4,72} & 4,83 \\
\hline & 6 & "Mi deseo de aprender se ve propiciado". & & 4,34 & 1,51 & 6 & & 4,81 & 1,32 & 6 & & 4,61 \\
\hline \multirow{2}{*}{$\begin{array}{l}\text { Interacción con el } \\
\text { grupo clase }\end{array}$} & 7 & "Las actividades desarrolladas fomentan la interacción con el grupo en clase". & \multirow{2}{*}{4,71} & 5,07 & 1,18 & 6 & \multirow{2}{*}{4,97} & 4,88 & 1,31 & 6 & \multirow{2}{*}{4,63} & 4,63 \\
\hline & 8 & "Los temas tratados me hacen plantearme preguntas". & & 4,34 & 1,47 & 5 & & 5,05 & 1,23 & 6 & & 4,62 \\
\hline \multirow{2}{*}{$\begin{array}{l}\text { Atención Individual } \\
\text { al Alumno }\end{array}$} & 9 & "Me siento respetado en mis opiniones". & \multirow{2}{*}{4,94} & 5,04 & 1,18 & 6 & \multirow{2}{*}{5,40} & 5,44 & 0,98 & 6 & \multirow{2}{*}{4,95} & 5,07 \\
\hline & 10 & "Me siento acompañado por el profesor". & & 4,85 & 1,37 & 6 & & 5,35 & 1,10 & 6 & & 4,83 \\
\hline \multirow{2}{*}{ Evaluación } & 11 & "Me he sentido exigido adecuadamente por este profesor". & \multirow{2}{*}{4,59} & 4,68 & 1,34 & 6 & \multirow{2}{*}{5,04} & 5,20 & 1,12 & 6 & 4.67 & 4,77 \\
\hline & 12 & "El método de evaluación permite reflejar lo que he aprendido". & & 4,5 & 1,43 & 5 & & 4,87 & 1,32 & 6 & & 4,60 \\
\hline Resultados & 13 & "Recibo feedback significativo que me permite mejorar". & 4.51 & 4,67 & 1,38 & 6 & 5.00 & 5,14 & 1,15 & 6 & 4,71 & 4,64 \\
\hline Kesuntados & 14 & "Aprendo cursando esta asignatura". & 4,51 & 4,35 & 1,57 & 6 & 5,00 & 4,86 & 1,40 & 6 & & 4,78 \\
\hline Cumplimiento & 15 & $\begin{array}{l}\text { "El profesor es puntual en sus compromisos (asistencia a clase, entrega de } \\
\text { notas...)". }\end{array}$ & 4,95 & 4,91 & 1,34 & 6 & 5,28 & 5,22 & 1,24 & 6 & 5,04 & 5,06 \\
\hline & 16 & "Es accesible en la atención al alumno (tutorias, correos, etc....)". & & 5 & 1,24 & 6 & & 5,34 & 1,09 & 6 & & 5,02 \\
\hline & 17 & $\begin{array}{l}\text { "Desde una consideración general, evalúa globalmente: La labor docente de este } \\
\text { profesor como facilitador de tu aprendizaje". }\end{array}$ & & 4,75 & 1,3 & 6 & & 5,20 & 1,09 & 6 & 4,73 & 4,81 \\
\hline Items-Criterio & 18 & $\begin{array}{l}\text { "Si pudieras, en qué grado te volverias a matricular en otra asignatura impartida } \\
\text { por este profesor". }\end{array}$ & 4,63 & 4,49 & 1,58 & 6 & 5,12 & 5,03 & 1,37 & 6 & & 4,64 \\
\hline & & MEDIA GLOBAL & 4,69 & 4,69 & 1,37 & 5,83 & 5,13 & 5,13 & 1,17 & 6,00 & 4,77 & 4,77 \\
\hline
\end{tabular}

Fuente: Crespí, 2018, p. 222 y 228.

Observando los datos de la tabla anterior, podemos decir, que esta asignatura, resulta, en términos globales muy bien valorada por los alumnos. Si observamos la media de la evaluación de todas las asignaturas de la universidad que es de 4,77, y la de las mentorías que es de 5,13 , podemos decir que estas superan la valoración media de la universidad en 0,36 puntos. No obstante, los alumnos parecen valorar mejor la parte de mentorías que la del aula, que tiene una media global de 4,69.

Por otro lado, la evaluación cualitativa nos indica que los alumnos valoran y agradecen especialmente el espacio de desarrollo personal que se les facilita a través de la asignatura de HCP.

En términos de significatividad, y a través del estadístico "t de student", (alpha 0,05) pudimos comprobar que las valoraciones de los alumnos de la docencia recibida en el aula, en la asignatura de HCP eran altas y no diferentes de las valoraciones de otras asignaturas, mientras que si resultaron significativamente superiores las valoraciones de la mentorías, como parte de la misma asignatura. 


\section{Conclusiones}

Dentro de la misión de la universidad en general y en particular de la UFV, y aludiendo a la necesidad detectada tanto del EEES como del ámbito empresarial, hemos propuesto la asignatura HCP.

Asignatura cuyo objetivo es el de acompañar a sus alumnos de primero en su respuesta a su vocación, aquí y ahora; de modo que puedan responder a ser quiénes están llamados a ser. Para lograr dichos objetivos, y ponerles en su propio camino de madurez, excelencia y plenitud, HCP articula el desarrollo de las competencias personales. Competencias transversales fundamentales en su desarrollo personal: cognitivas, intra e interpersonales.

Por tanto, HCP pretende que el alumno pueda reflexionar acerca de quién es y de quién está llamado a ser, desarrollando las competencias personales que le permitan dar lo mejor de sí mismo en cada momento. Para ello, el alumno es acompañado en el aula por sus compañeros y profesor; y en la mentoría, por su mentor.

Por último, la valoración de esta asignatura, y muy especialmente la de las mentorías, como espacio de desarrollo personal, es muy positiva, tanto cuantitativa como cualitativamente.

\section{Referencias}

Alles, M. A. (2008). Desempeño por competencias: evaluación de $360^{\circ}$ (2 ed.). Buenos Aires: Granica.

Benito, A., y Cruz, A. (2006). Nuevas claves para la Docencia Universitaria en el Espacio europeo de Educación Superior. Madrid: Narcea.

Crespí, P. (2018). La necesidad de una formación en competencias personales en la universidad. Diseño y evaluación de un programa de formación. Tesis Doctoral. Universidad Complutense de Madrid. Facultad de Educación, Madrid.

Crespí, P. (2019). La necesidad de una formación en competencias personales transversales en la universidad. Diseño y evaluación de un programa de formación. Madrid: Fundación Universitaria Española.

Crespí, P., y García Ramos, J.M. (2019). Diseño y evaluación de un programa de formación en competencias personales transversales en la universidad. En J. Murillo (Presidencia), Congreso Internacional de Investigación Educativa: Investigación comprometida para la transformación social. AIDIPE, Facultad de Formación de Profesorado y Educación de la Universidad Autónoma de Madrid, Madrid.

García Ramos, J. M. (1991). La formación integral: objetivo de la Universidad. Algunas reflexiones sobre la educación en la Universidad. Revista Complutense de Educación, 2, 323-335. 
Habilidades y Competencias de la Persona. Una asignatura para el desarrollo de las competencias personales

García Ramos, J. M. (2017). Misión de la Universidad en los tiempos de la Postverdad. Conferencia inaugural del curso 2017-2018. Universidad Católica de Ávila.

González Ariza, A. L. (2017). Métodos de compensación basados en competencias (3 ed.). Área metropolitana de Barranquilla: Editorial Universidad del Norte.

González, J., y Wagenaar, R. (2003). Tuning Educational Estructures in Europe. Informe Final Fase Uno. Bilbao: Universidad de Deusto.

González, J., y Wagenaar, R. (2006). Tuning Educational Structures in Europe II. La contribución de las universidades al Proceso de Bolonia. Bilbao: Universidad de Deusto.

Gutiérrez Tobar, E. (2010). Competencias gerenciales: habilidades, conocimientos, aptitudes. Bogotá: Ecoe Ediciones.

Jaspers, K. (2013). La idea de la universidad. Navarra: Ediciones Universidad de Navarra.

Moncada, J. S. (2008). La universidad: un acercamiento histórico filosófico. Ideas y valores, 57(137), 131-148.

Nuestra misión hoy. (2016). Madrid: Editorial UFV.

Ortega y Gasset, J. (2004). Misión de la universidad y otros ensayos sobre educación y pedagogía. Madrid: Alianza Editorial.

Poblete Ruiz, M., y García Olalla, A. (2007). Desarrollo de competencias y Créditos transferibles. Experiencia multidisciplinar en el contexto universitario/Poblete Ruiz y García Olalla (Coords.). Bilbao: Universidad de Deusto.

Pérez-Díaz, V. (2010). Universidad, ciudadanos y nómadas. Oviedo: Ediciones Nobel.

Villardón-Gallego, L. (2015). Competencias genéricas en educación superior. Metodologías específicas para su desarrollo/Villardón-Gallego (Coord.). Madrid: Narcea. 